6 - ORIGINAL ARTICLE

ISCHEMIA/REPERFUSION

\title{
Parecoxib reduces renal injury in an ischemia/reperfusion model in rats ${ }^{1}$
}

\author{
José Pedro Calistro Neto', Rômulo da Costa Torres, Giovanna Maria Gonçalves ${ }^{\mathrm{II}}$, Leopoldo Muniz da Silva ${ }^{\mathrm{III}}$, Maria Aparecida \\ Custódio Domingues ${ }^{\mathrm{IV}}$, Norma Sueli Pinheiro Módolov, Guilherme Antonio Moreira de Barros ${ }^{\mathrm{VI}}$
}

DOI: http://dx.doi.org/10.1590/S0102-865020150040000006

IFellow Master degree, Postgraduate Program in Anesthesiology, Botucatu Medical School, Sao Paulo State University (UNESP), Brazil. Conception, design, intellectual and scientific content of the study.

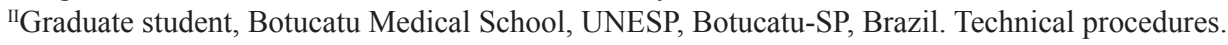

IIIMD, Division of Anesthesiology, Botucatu Medical School, UNESP, Botucatu-SP, Brazil. Acquisition and interpretation of data, statistical analysis.

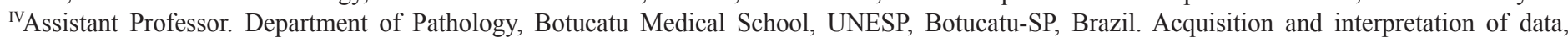
histological examinations.

${ }^{\vee}$ Full Professor, Department of Anesthesiology, Botucatu Medical School, UNESP, Botucatu-SP, Brazil. Critical revision.

${ }^{v 1}$ Assistant Professor, Department of Anesthesiology, Botucatu Medical School, UNESP, Botucatu-SP, Brazil. Manuscript writing, supervised all phases of the study.

\section{ABSTRACT}

PURPOSE: To evaluate the effect of parecoxib (an NSAID) on renal function by measuring plasma NGAL (serum neutrophil gelatinaseassociated lipocalin) levels in an induced-ischemia rat model.

METHODS: Forty male Wistar rats were randomly assigned to one of four groups: Ischemia (I), Ischemia/parecoxib (IP), No-ischemia (NI), and No-ischemia/parecoxib (NIP). Body weight, mean arterial pressure, heart rate, body temperature, NGAL levels, and renal histology were compared across groups.

RESULTS: The Ischemia (I) group, which did not receive parecoxib, showed the highest NGAL levels ( $\mathrm{p}=0.001$ ), while the IP group, which received the medication, had NGAL levels similar to those of the non-ischemic (NI and NIP) groups.

CONCLUSION: Parecoxib resulted in renal protection in this experimental model.

Key words: Parecoxib. Ischemia. Reperfusion. Kidney. Rats. 


\section{Introduction}

Nonsteroidal anti-inflammatory drugs (NSAIDs) are the most commonly-used class of drugs in the world ${ }^{1}$ and are used to treat a number of clinical symptoms, including acute pain ${ }^{2}$. NSAIDs inhibit the activity of cyclooxigenases (COXs) and are classified as selective (selective COX-2 inhibitors) or non-selective (COX-1 and COX-2 inhibitors), according to their mechanism of action $^{3}$. Thromboxanes and prostanoids are products of COX-1 and COX-2, respectively, and are involved in the maintenance of homeostasis of different organs and systems (e.g., gastrointestinal tract, kidney, heart, lung, brain, and platelets $)^{4}$.

COX-1 is also called a constitutive enzyme and is involved in cell function, regeneration and repair, whereas COX-2, which is called an inducible enzyme, depends on an inflammatory process or the malfunction of the aforementioned organs and systems $\mathrm{s}^{3,4}$. However, the detection of COX-2 in the kidneys under normal conditions or under conditions of impaired renal blood flow (RBF) and glomerular filtration rate (GFR) has attracted the attention of researchers to the role of these drugs in maintaining renal function ${ }^{4-6}$.

Parecoxib sodium is the only COX-2-selective NSAID available in injectable form and approved for clinical use and has comparable efficacy to that of non-selective NSAIDs ${ }^{7}$. However, administration of parecoxib may affect renal function in an unknown way, especially in situations of diminished renal perfusion such as dehydration, hemorrhage, heart failure, low-salt diets, and stroke $\mathrm{e}^{3,8,9}$. Renal function can be monitored using the neutrophil gelatinase-associated lipocalin (NGAL) biomarker, whose expression is induced by renal epithelial injury. In fact, NGAL is one of the earliest markers of renal injury after ischemia or nephrotoxic injury in animal models ${ }^{10}$.

There is no consensus on the effect of parecoxib on renal function. For instance, Patel et al. ${ }^{11}$ used the concentration of creatinine in ischemia/reperfusion models in rats and showed that parecoxib increases renal dysfunction and injury associated with this form of renal stress. Conversely, Hauser et al. ${ }^{12}$ showed that parecoxib attenuates renal function impairment during porcine suprarenal aortic cross-clamping.

This study aimed to evaluate the effects of parecoxib on renal function by measuring plasma NGAL levels in an inducedischemia model in rats and histologically evaluate possible lesions.

\section{Methods}

The study was approved by the UNESP Ethics Committee on Animal Use.
This is a prospective, randomized blind study. Forty male Wistar rats weighing more than $250 \mathrm{~g}$ were used. The sex of the animals, the experimental model, and the sample size were determined based on a previous study from our group that used the same methodology ${ }^{13}$. The animals were assigned to one of four experimental groups, with ten animals per group: Ischemia (I), Ischemia/parecoxib (IP), No-ischemia (NI), and No-ischemia/ parecoxib (NIP) (Table 1).

TABLE 1 - Experimental groups: Ischemia (I), Ischemia/ parecoxib (IP), No-ischemia (NI), and No-ischemia/parecoxib (NIP).

\begin{tabular}{ccccc}
\hline Group & $\begin{array}{c}\text { Inhalational } \\
\text { anesthesia }\end{array}$ & $\begin{array}{c}\text { Right } \\
\text { nephrectomy }\end{array}$ & $\begin{array}{c}\text { Left } \\
\text { ischemia }\end{array}$ & Parecoxib \\
\cline { 2 - 5 } I & + & + & + & - \\
IP & + & + & + & + \\
NI & + & + & - & - \\
NIP & + & + & - & + \\
\hline
\end{tabular}

The animals were anesthetized with isoflurane, intubated, and kept under mechanical ventilation. Body temperature was kept between $36^{\circ}$ and $37^{\circ} \mathrm{C}$ using a heating pad, and mean arterial pressure (MAP), heart rate (HR), and body temperature were continuously monitored using a multi-parameter monitor.

Animals were catheterized in the arterial and venous accesses under local anesthesia of the bilateral cervical region with $0.3 \mathrm{ml}$ of $0.25 \%$ bupivacaine and depending on the experimental group, received either $20 \mathrm{mg} / \mathrm{kg}$ parecoxib or $2 \mathrm{ml} / \mathrm{kg}$ saline solution, intravenously. The doses of parecoxib were determined according to the calculation recommended by the Food and Drug Administration (FDA) ${ }^{14}$. Volume replacement was performed with continuous infusion of $5 \mathrm{ml} / \mathrm{h}$ of Ringer's lactate solution (RLS).

After infiltration of the skin and subcutaneous tissue with $0.7 \mathrm{ml}$ of $0.25 \%$ bupivacaine, median laparotomy was performed, followed by right nephrectomy. Next, the left renal artery was clamped for $30 \mathrm{~min}$.

Blood samples were collected at the following times: T1 (immediately after arterial access), T2 (5 min after clamping of the left renal artery), T3 (20 min after reperfusion of the left kidney) and T4 (24 h after the beginning of the procedure). At each time point, $1 \mathrm{ml}$ samples of blood were taken and immediately replaced with the injection of $2 \mathrm{ml}$ RLS.

Blood samples were centrifuged and the supernatant was stored in a freezer at $-80{ }^{\circ} \mathrm{C}$. NGAL levels were measured using ELISA (enzyme-linked immunosorbent assay) with a Rat NGAL ELISA kit (Kit 046, BioPorto Diagnostics, Hellerup, Denmark). 
This immunoenzyme assay detects the specific plasma antibody by antigen-antibody interaction.

Right and left kidneys were stored separately in DuboscqBrasil solution for $24 \mathrm{~h}$ and then transferred to $70 \%$ ethyl alcohol. The Duboscq-Brasil solution is used to preserve and fixate renal structures and increase the reliability of the histological analysis. Histological sections were stained with hematoxylin-eosin and evaluated with a light microscope for percentage of tubular necrosis by a pathologist who was blind to which experimental groups the samples belonged to, and classified using the scale proposed by Park et al. ${ }^{13}$ (Table 2). The following microscopic criteria were used to determine the necrosis of tubular cells: pyknosis, karyorrhexis or karyolysis, and chromatolysis.

TABLE 2 - Cell damage scores attributed to histological specimens.

\begin{tabular}{ccc}
\hline Degree of injury & Type of injury & $\%$ of injury \\
\hline 0 & no lesion & 0 \\
1 & mild & $<10$ \\
2 & moderate & $10-25$ \\
3 & moderately severe & $25-50$ \\
4 & severe & $50-75$ \\
5 & very severe & $>75$ \\
\hline
\end{tabular}

Adapted from Park et al. ${ }^{13}$.

Data were normally distributed and were expressed as means \pm standard deviations. We used analysis of variance (ANOVA) followed by the Tukey's test at $p<0.05$ to compare mean body weight, MAP, HR, body temperature, and NGAL levels between experimental groups and the Friedman's test to compare these variables within a group at different times. Renal histology was scored and renal lesions of right and left kidneys were expressed as medians (25-75\% percentiles) and compared using the Kruskal-Wallis one-way analysis of variance followed by the Dunn's post-hoc test at $\mathrm{p}<0.05$. The Mann-Whitney test was used for intragroup comparison of renal histology scores. We used the Fisher's exact test to compare the frequency of renal lesions between the Ischemia (I) and Ischemia/parecoxib (IP) groups. All analyses were performed using the Stata/SE 9.0 software for Windows (Stata Corporation, College Station, Texas, USA). The significance level was set at $\mathrm{p}<0.05$.

\section{Results}

Mean body weight was higher in the I and NI groups than in the IP and NIP groups ( $<$ 0.05). Mean arterial pressure (MAP) also differed significantly between groups: at T1, MAP was higher in the I group than in the NIP group $(\mathrm{p}=0.03)$, whereas at T2, MAP was lower in the IP than in the NIP group $(\mathrm{p}=0.01)$; at T3, MAP values were highest in the I group $(p=0.001)$. No significant differences in MAP were observed between the I and NI groups at any times, but MAP values were higher in T1 than in T2 and $\mathrm{T} 3$ in the IP group $(\mathrm{p}=0.007)$ and in T2 than in T1 and T3 in the NIP group ( $\mathrm{p}=0.0002)$ (Figure 1). In addition, no significant differences in heart rate and body temperature were observed between groups.

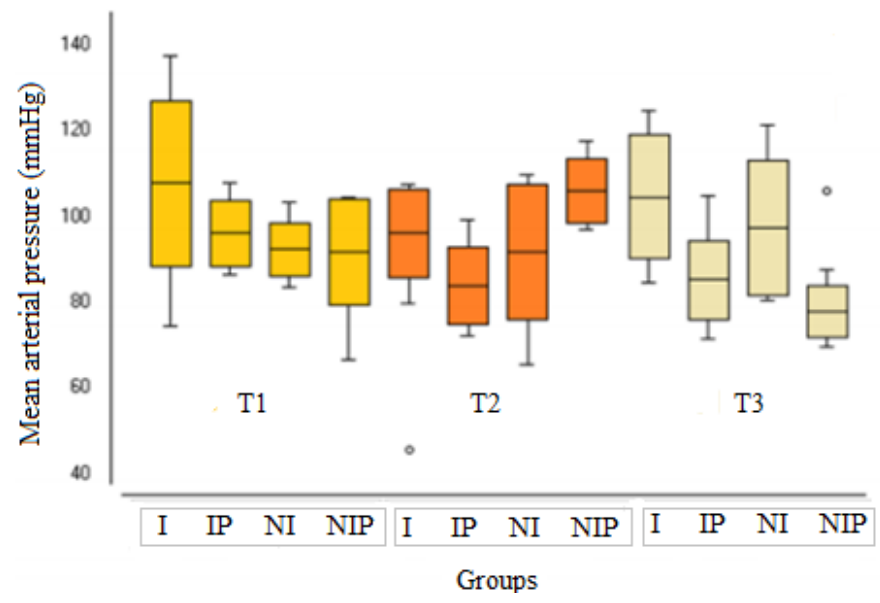

FIGURE 1 - Mean arterial pressure (MAP) of male Wistar rats in the Ischemia (I), Ischemia/parecoxib (IP), No-ischemia (NI), and Noischemia/parecoxib (NIP) groups at different times. The lines in the box represent the means (middle line) and standard deviations (upper and lower lines). Comparison between groups: T1: I > NIP $(p=0.03)$; T2: IP $<$ NIP $(\mathrm{p}=0.01)$; T3: I $>$ IP, I $>$ NIP, NI $>$ NIP $(\mathrm{p}=0.001)$. Comparison between times: I: $(\mathrm{p}=0.12)$; IP: T1 > T2, T1 > T3 (p=0.007); NI ( $\mathrm{p}=$ 0.78); NIP: $\mathrm{T} 1<\mathrm{T} 2, \mathrm{~T} 2>\mathrm{T} 3(\mathrm{p}=0.0002)$.

NGAL serum levels differed significantly between groups and times (Table 3). NGAL levels were significantly higher at T4 relative to the other time points in all groups: $\mathrm{I}(\mathrm{p}=0.006)$, IP $(p<0.001)$, NI $(p<0.001)$, and NIP $(p<0.001)$. In addition, no differences in NGAL levels were observed between groups at T1 and T2. Conversely, NGAL levels were significantly higher in the I group than in the IP, NI, and NIP groups at T3 $(p=0.03)$ and T4 $(\mathrm{p}=0.002)($ Table 3$)$. 
TABLE 3 - NGAL levels (picograms per milliliter [pg/ $\mathrm{ml}$, mean $\pm \mathrm{sd}$ ) of male Wistar rats in the Ischemia (I), Ischemia/ parecoxib (IP), No-ischemia (NI), and No-ischemia/parecoxib (NIP) groups at different times.

\begin{tabular}{ccccc}
\hline \multirow{2}{*}{ Group } & \multicolumn{4}{c}{ Time } \\
\cline { 2 - 5 } & $\mathrm{T} 1$ & $\mathrm{~T} 2$ & $\mathrm{~T} 3$ & $\mathrm{~T} 4$ \\
\hline $\mathrm{I}$ & $15.23 \pm 6.4$ & $32.43 \pm$ & $37.54 \pm$ & $1186.63 \pm$ \\
& & 35.52 & 38.64 & 1574.77 \\
$\mathrm{IP}$ & $11.72 \pm$ & $10.68 \pm 5.7$ & $12.63 \pm 5.48$ & $254.56 \pm$ \\
& 5.45 & & & 103.69 \\
NI & $12.44 \pm$ & $23.51 \pm$ & $15.99 \pm 5.21$ & $311.05 \pm$ \\
& 5.51 & 34.86 & & 163.2 \\
NIP & $11.80 \pm$ & $10.02 \pm 2.91$ & $12.84 \pm 2.55$ & $295.63 \pm$ \\
& 3.42 & & & 78.37 \\
\hline
\end{tabular}

Comparison between times: I: T1 $<\mathrm{T} 4, \mathrm{~T} 2<\mathrm{T} 4, \mathrm{~T} 3<\mathrm{T} 4(\mathrm{p}=0.006)$; IP: $\mathrm{T} 1<\mathrm{T} 4$, T2 $<$ T4, T3 $<$ T4 $(\mathrm{p}<0.001) ; \mathrm{NI}: \mathrm{T} 1<\mathrm{T} 4 ; \mathrm{T} 2<\mathrm{T} 4, \mathrm{~T} 3<\mathrm{T} 4(\mathrm{p}<0.001) ; \mathrm{NIP}: \mathrm{T} 1$ $<\mathrm{T} 4, \mathrm{~T} 2<\mathrm{T} 4, \mathrm{~T} 3<\mathrm{T} 4(\mathrm{p}<0.001)$. Comparison between groups: $\mathrm{T} 1(\mathrm{p}=0.56) ; \mathrm{T} 2$ $(\mathrm{p}=0.17) ; \mathrm{T} 3: \mathrm{I}>\mathrm{IP}, \mathrm{NI}$, and NIP $(\mathrm{p}=0.03) ; \mathrm{T} 4: \mathrm{I}>\mathrm{IP}, \mathrm{NI}$, and NIP $(\mathrm{p}=0.002)$.

For the histological analysis, right kidneys in all experimental groups had a score of zero for acute tubular necrosis. Animals in the NI and NIP groups, which had not received renal ischemia-reperfusion but only systemic injection of antiinflammatory drugs, also had a score of zero for acute tubular necrosis. The left kidneys of animals in the I and IP groups, which had been subjected to ischemia-reperfusion and administration of parecoxib (IP), had varying scores of acute tubular necrosis. Lesions were more frequent in rats in the I group, in which seven animals had lesions, with scores ranging from 1-4 (mild to moderate lesions), and less frequent in the IP group, in which only one animal had a renal injury with a score of 2 (mild lesion). Thus, tubular lesions were more frequent in the I group than in the IP, NI, and NIP groups (Figure 2).

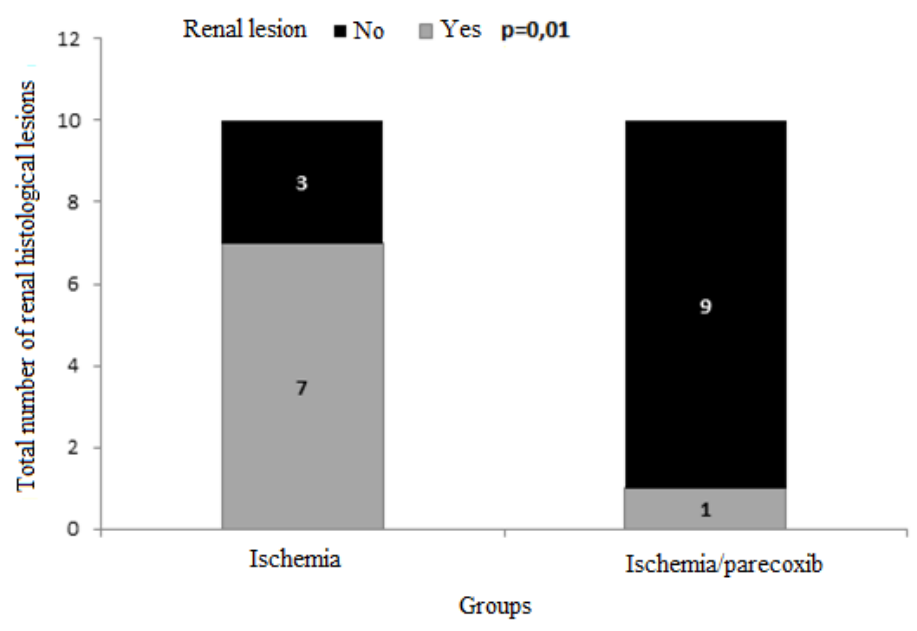

FIGURE 2 - Total number of renal histological lesions in the left kidney of male Wistar rats in the Ischemia (I) and Ischemia/parecoxib (IP) groups (Fisher's exact test). The number of histological renal lesions was lower in the IP than in the I group $(\mathrm{p}=0.01)$.
The medians of lesion severity and intensity were higher in the I and IP groups than in the NI and NIP groups. Lesion severity ranged from mild to severe (Table 4).

TABLE 4 - Lesion severity in right and left kidneys of male Wistar rats in the Ischemia (I), Ischemia/parecoxib (IP), Noischemia (NI), and No-ischemia/parecoxib (NIP) groups.

\begin{tabular}{|c|c|c|}
\hline \multirow[t]{2}{*}{ Group } & \multicolumn{2}{|c|}{ Kidney } \\
\hline & Right & Left \\
\hline I & $0(0.00)$ & $1(0.25-2)[4]$ \\
\hline IP & $0(0.00)$ & $0(0.00)$ \\
\hline NI & $0(0.00)$ & $0(0.00) \quad[0]$ \\
\hline NIP & $0(0.00)$ & $0(0.00) \quad[0]$ \\
\hline
\end{tabular}

Values expressed as medians $(25-75 \%$ percentiles) [total range]. Kruskal-Wallis one-way ANOVA - Dunn's post-hoc test: Left kidney: I > IP, I> NIP $(p=0.01)$; Right kidney: n.s. Intragroup comparison (Mann-Whitney test): I $(p=0.004), \operatorname{IP}(p=0.70)$, NI $(p=1.00), \operatorname{NIP}(p=1.00)$.

\section{Discussion}

In this study, we used the ischemia/reperfusion model developed for kidney function and injury studies at the Department of Anesthesiology, Botucatu Medical School. This model consists of right nephrectomy in all animals, followed by randomization of the animals into four groups of 10 animals per group: Ischemia, Ischemia + drug, Non-ischemia, and Non-ischemia + drug. Thus, parecoxib was tested in two groups, in the presence and absence of I/R (IP and NIP groups), and NGAL levels were measured in all groups at four times to evaluate renal function.

Nephrectomy followed by ischemia/reperfusion may trigger mechanisms of renal preconditioning, reducing histological lesions and preserving or facilitating recovery of renal function in the ischemic kidney. For instance, animals that had not been nephrectomized prior to ischemia/reperfusion had oliguric renal failure, whereas animals that had been nephrectomized prior to $\mathrm{I} / \mathrm{R}$ developed polyuric renal failure with better prognosis and recovery of renal function. The incidence of renal lesions in the ischemic kidney was also higher in non-nephrectomized animals. Plasma renin activity and renin content in the post-ischemic kidney increased significantly in nephrectomized rats. Conversely, the levels of thromboxane A 2 and endothelin, which are responsible for renal vasoconstriction, were higher in non-nephrectomized rats ${ }^{17}$.

Renal susceptibility to ischemic injury is directly related to body temperature. Thus, maintaining body temperature within a constant range and recording it is essential for the validation of results, especially in ischemic models. In the absence of ischemic stress, temperatures above $38^{\circ} \mathrm{C}$ do not alter renal morphology and 
function. However, hyperthermia has been shown to cause severe lesions in ischemia-reperfusion models. Conversely, temperatures below $35^{\circ} \mathrm{C}$ provided a protective effect, resulting in mild, reversible lesions and renal recovery $24-48 \mathrm{~h}$ after ischemic injury. In our study, all animals were normothermic, with temperatures ranging between 36 and $37^{\circ} \mathrm{C}$, a temperature range that favors a rapid recovery from ischemic renal injury. Thus, no significant differences in body temperature were observed during the study. Similarly, heart rate did not differ significantly between groups ${ }^{15,16}$.

The right nephrectomy and stable temperature resulted in similar ischemic preconditioning and susceptibility to renal injury in all groups, which reinforces our choice of using this model to evaluate the influence of parecoxib on renal function in experimentally simulated simulations that are frequent in clinical practice such as hemorrhage, dehydration, heart failure, surgery performed under extracorporeal circulation, and shock.

Mean body weight was higher for animals in the I and NI groups than in the IP and NIP groups. However, we believe that this weight difference between groups had no effect on data collection because we used the human-equivalent parecoxib dosage based on FDA guidelines for animal testing. Additionally, all animals weighed more than $250 \mathrm{~g}$, which was the minimum weight for inclusion in the study. Moreover, volume replacement was based on the individual weight of each animal ${ }^{14}$.

The effects of NSAIDs on blood pressure have been investigated in several experimental trials and in heterogeneous populations ${ }^{18,19}$. COX-2-derived prostanoids such as PGE-2 and PGI2 , which act on renal tubules and ducts and on the macula densa, respectively, modulate the resorption of sodium and water and stimulate the production and release of renin, which acts as a potent systemic and renal vasoconstrictor ${ }^{3,20}$. This action increases the resorption of sodium/ water and the secretion of potassium and maintains the glomerular filtration rate (GFR) and renal homeostasis, especially in situations of hemodynamic impairment and consequent renal injury ${ }^{21}$. Additionally, COX-1 prostanoids such as thromboxane also act as vasoconstrictors and increase the resorption of sodium and water ${ }^{3}$.

NSAIDs are well-tolerated by individuals without impaired renal function and who do not depend on the COX system to maintain renal hemodynamics. However, NSAIDs should be used with caution in older individuals and in those with congestive heart failure, liver cirrhosis, low-salt diets, or in situations of hypovolemia, because these individuals are dependent on the mechanisms described above to maintain adequate $\mathrm{GFR}^{3,20}$. The use of parecoxib after coronary-artery bypass grafting was associated with an increased incidence of cardiovascular events and should be avoided in patients undergoing this type of procedure?.
Blood pressure patterns were similar in the groups that received parecoxib (IP and NIP) and lower than in groups that did not receive parecoxib (I and NI). Also, treated animals had the lowest blood pressure levels at T3, regardless of whether they had been exposed to I/R injury.

The reduction in blood pressure was also observed in another study about the effect of parecoxib on renal function. The hypotensive effect in treated animals, combined with the diminished creatinine clearance and worse histology were associated with a negative effect of parecoxib on renal function, which was attributed to the non-activation of the renin-angiotensin-aldosterone system secondary to the inhibition of COX-2 by parecoxib. The authors also argued that COX-2 is of great importance for renal dynamics, especially in situations of imminent risk or renal injury ${ }^{11}$.

A study that evaluated the effects of intrarenal administration of parecoxib during suprarenal aortic crossclamping in pigs found that parecoxib attenuated renal impairment measured by kidney creatinine clearance and improved renal lactate balance. Additionally, no effects on the cardiovascular system of treated animals were observed, and the authors concluded that intrarenal parecoxib infusion positively affected kidney function ${ }^{12}$.

The reduction in blood pressure in the groups that received parecoxib was not pronounced and the blood pressure levels recorded were able to maintain RBF and GFR and not significantly increase NGAL serum levels.

NGAL serum levels were lowest at $\mathrm{T} 1$ and highest at T4 in all groups. In addition, NGAL levels were significantly higher in the Ischemia group (I), which was not treated with parecoxib, than in the IP, NI, and NIP groups. Conversely, at T4, NGAL levels in the IP group were similar to those in the NI and NIP groups, which had not been subjected to I/R injury, and lower than in the I group.

NGAL levels in the I group were higher at T3 and T4, confirming the precocity of this biomarker in serum measures only $50 \mathrm{~min}$ after injury (30 min of renal ischemia and $20 \mathrm{~min}$ of renal reperfusion). The use of parecoxib prior to $I / R$ injury in the IP group was solely responsible for the lower NGAL levels in this group. Moreover, the use of parecoxib in the NIP group did not result in increased NGAL levels.

A similar study about the effects of indomethacin, a non-selective NSAID, and rofecoxib, a selective NSAID, showed that both NSAIDs were associated with improved renal function, as measured by blood urea levels. The authors also observed a reduction in proinflammatory cytokine levels and fewer histological lesions, especially in animals treated with rofecoxib. Part of the renal injury secondary to ischemia/reperfusion is a 
consequence of the inflammatory response with involvement of COX-2 and prostaglandins, in addition to the production and secretion of chemokines, cytokines (IL-1, IL-6, IL-19, and TNF- $\alpha$ ), and reactive oxygen species, gene activation, and cell infiltration. The use of NSAIDs inhibited part of this response, resulting in renal protection ${ }^{22}$.

No signs of histological lesions were observed in previously nephrectomized right kidneys and left kidneys that had not been subjected to ischemia (NI and NIP groups). Histological lesions were observed only in the Ischemia (I) and Ischemia/ parecoxib (IP) groups, and were more frequent in animals in the I group. The use of parecoxib was solely responsible for the lower frequency of lesions in the IP than in the I group. The medians of cell damage scores did not differ between the I and IP groups and were significantly higher in these groups than in the NI and NIP groups. Thus, parecoxib administration does not appear to determine lesion intensity in our experimental model.

\section{Conclusion}

Parecoxib provided renal protection to animals subjected to ischemia-reperfusion and its administration in non-ischemic animals was not responsible for renal injury based on NGAL levels and histological analysis.

\section{References}

1. Singh G, Triadafilopoulos G. Epidemiology of NSAID induced gastrointestinal complications. J Rheumatol Suppl. 1999 Apr;56:1824. Review. PMID: 10225536.

2. Kummer CL, Coelho TCRB. Anti-inflamatórios não esteroidais inibidores da ciclooxigenase-2 (COX-2): aspectos atuais. Rev Bras Anestesiol. 2002 Jul;52(4):498-512. PMID: 19479115.

3. Harris RC. COX-2 and the kidney. J Cardiovasc Pharmacol. 2006;47 Suppl 1:S37-42. Review. PMID: 16785827.

4. Dirig DM, Isakson PC, Yaksh TL. Effect of COX-1 and COX2 inhibition on induction and maintenance of carrageenanevoked thermal hyperalgesia in rats. J Pharmacol Exp Ther. 1998 Jun;285(3):1031-8. PMID: 9618405.

5. Brater DC, Harris C, Redfern JS, Gertz BJ. Renal effects of COX2-selective inhibitors. Am J Nephrol. 2001 Jan-Feb;21(1):1-15. Review. PMID: 11275626.

6. Whelton A. Renal and related cardiovascular effects of conventional and COX-2-specific NSAIDs and non-NSAID analgesics. Am J Ther. 2000 Mar;7(2):63-74. Review. PMID: 11319575.

7. Cheer SM, Goa KL. Parecoxib (parecoxib sodium). Drugs. 2001;61(8):1133-41. PMID: 11465874.

8. Zhang J, Ding EL, Song Y. Adverse effects of cyclooxygenase 2 inhibitors on renal and arrhythmia events: meta-analysis of randomized trials. JAMA. 2006;Oct;296(13):1619-32. PMID: 16968832.

9. Nussmeier NA, Whelton AA, Brown MT, Langford RM, Hoeft A, Parlow JL, Boyce SW, Verburg KM. Complications of the COX-2 inhibitors parecoxib and valdecoxib after cardiac surgery. N Engl J
Med. 2005 Mar;352(11):1081-91. PMID: 15713945.

10. Mishra J, Dent C, Tarabishi R, Mitsnefes MM, Ma Q, Kelly C, Ruff SM, Zahedi K, Shao M, Bean J, Mori K, Barasch J, Devarajan P. Neutrophil gelatinase-associated lipocalin (NGAL) as a biomarker for acute renal injury after cardiac surgery. Lancet. 2005 Apr;365(9466):1231-8. PMID: 15811456.

11. Patel NS, Cuzzocrea S, Collino M, Chaterjee PK, Mazzon E, Britti $\mathrm{D}$, Yaqoob MM, Thiemermann C. The role of cycloxygenase-2 in the rodent kidney following ischaemia/reperfusion injury in vivo. Eur J Pharmacol. 2007 May;562(1-2):148-54. PMID: 17343844.

12. Hauser B, Froba G, Bracht H, Strater J, Chkhouta AB, Vassilev D, Schoaff MJ, Huber-Lang M. Effects of intrarenal administration of the cox-2 inhibitor parecoxib during porcine suprarenal aortic crossclamping. Shock. 2005 Nov;24(5):476-81. PMID: 16247335.

13. Park Y, Hirose R, Dang K, Xu F, Behrends M, Tan V, Roberts JP, Niemann CU. Increased severity of renal ischemia-reperfusion injury with venous clamping compared to arterial clamping in a rat model. Surgery. 2008 Feb;143(2):243-51. doi: 10.1016/j. surg.2007.07.041.

14. Food and Drug Administration. Guidance for industry: estimating the maximum safe starting dose in intial clinical trial for therapeuthics in adult health volunteers. Silver Spring: FDA; 2005.

15. Zager RA, Altschuld R. Body temperature: an important determinant of severity of ischemic renal injury. Am J Physiol. 1986 Jul;251(1 Pt 2):F87-93. PMID: 3728686.

16. Delbridge MS, Shrestha BM, Raftery AT, El Nahas AM, Haylor JL. The effect of body temperature in a rat model of renal ischemiareperfusion injury. Transplant Proc. 2007 Dec;39(10):2983-5. PMID: 18089305.

17. Islam CF, Mathie RT, Dinneen MD, Kiely EA, Peters AM, Grace PA. Ischaemia-reperfusion injury in the rat kidney: the effect of preconditioning. Br J Urol. 1997 Jun;79(6):842-7. PMID: 9202547.

18. Johnson AG. NSAIDs and increased blood pressure. What is the clinical significance? Drug Saf. 1997 Nov;17(5):277-89. Review. PMID: 9391772.

19. Wang JL, Cheng HF, Harris RC. Cyclooxygenase-2 inhibition decreases renin content and lowers blood pressure in a model of renovascular hypertension. Hypertension. 1999 Jul;34(1):96-101. PMID: 104068.

20. Brater DC, Harris C, Redfern JS, Gertz BJ. Renal effects of COX2-selective inhibitors. Am J Nephrol. 2001 Jan-Feb;21(1):1-15. PMID: 11275626.

21. Noroian G, Clive D. Cyclo-oxygenase-2 inhibitors and the kidney: a case for caution. Drug Saf. 2002;25(3):165-72. PMID: 11945113.

22. Feitoza CQ, Goncalves GM, Semedo P, Cenedeze MA, Pinheiro HS, Beraldo FC, dos Santos OF, Teixeira Vde P, dos Reis MA, Mazzali M, Pacheco-Silva A, Câmara NO. Inhibition of COX 1 and 2 prior to renal ischemia/reperfusion injury decreases the development of fibrosis. Mol Med. 2008 Nov-Dec;14(11-12):724-30. doi: 10.2119/2008-00064.

\section{Correspondence:}

Guilherme Antonio Moreira de Barros

Departamento de Anestesiologia

Faculdade de Medicina de Botucatu-UNESP

Distrito de Rubião Junior

Caixa Postal 530

18618-970 Botucatu - SP Brasil

Tel.: (55 14)3880-1411

barros@fmb.unesp.br 
Calistro Neto JP et al.

Received: Dec 16, 2014

Review: Feb 19, 2015

Accepted: Mar 18, 2015

Conflict of interest: none

Financial source: none

${ }^{1}$ Research performed at Experimental Laboratory of Anesthesiology, Botucatu Medical School, Sao Paulo State University (UNESP), Brazil.

Part of Master degree thesis, Postgraduate Program in Anesthesiology,

UNESP. Tutor: Guilherme Antonio Moreira de Barros. 